Journal of Social Sciences 8 (3): 435-446, 2012

ISSN 1549-3652

(C) 2012 Science Publications

\title{
A Preliminary Investigation of the Adversity Reading in Archaic WEI Passives
}

\author{
Pei-Jung Kuo and Jun-Yu Zhang \\ Department of Foreign Languages, \\ College of Humanities and Arts, National Chiayi University, \\ No.85, Wunlong Village, Minsyong Township, Chiayi County 621, Taiwan
}

\begin{abstract}
Problem statement: It is well-known that modern Chinese imposes such a requirement on the subject in the BEI passive, but in fact it does not hold tightly in Modern Chinese. Only 50-75\% of passives have the adversity reading on the subject. Approach: One approach to explain this phenomenon is to adopt the view of grammaticalization and trace the semantic change of the morpheme BEI. However, the majority of the passive construction in archaic Chinese is not the BEI passive, but the WEI construction. The Adversity reading in the WEI construction in archaic Chinese is examined as a pilot study in this study. Results: Our research result shows that in the book of Shi Ji, among 50 tokens, $78 \%$ of the WEI passive has the adversity reading. Conclusion: This thus indicates that our impression about the adversity reading imposed on passives is not an absolute requirement, no matter in modern or archaic Chinese. On the other hand, the investigation of the WEI...SUO construction indicates that there is a decline of the adversity reading. We therefore conclude that a follow-up study should be carried out to complete the whole picture of the investigation of the adversity reading in Chinese passives.
\end{abstract}

Key words: The BEI passive, the WEI (...SUO) passive, grammaticalization and language change

\section{INTRODUCTION}

Unlike passives in English, it is widely assumed that most passives in Chinese impose an adversity reading on the grammatical subject (Hsin, 1990; Liu et al., 2001; Tseng, 2004), as articulated by the authors of a recent textbook for teachers of Chinese as a Second Language, "In modern Mandarin Chinese, BEI constructions are employed mostly when the subject suffers from some damage or feels unhappy. Therefore its usage is very limited" (Liu et al., 2001, p.423) (the original excerpt from Liu et al. (2001) is as follows: "Zai xiandai hanyu Putonghua li, 「bei」ziju muqian duo yongyu dui zhuyu laishuo shi shousun-de huo bu yukuai-de qingxing, suoyi ta-de shiyong shi bijiao youxian-de。 (p. 423)). Hence, examples like (1a) and (1b), with a beneficiary reading on the subject, are considered ungrammatical. In contrast, the example in (2), which has an adversity/malificiary reading, is allowed (However, as pointed out by Liu et al. (2001), if a verbal complement is added after the verb ai ('love') in (3b), this sentence becomes acceptable in Chinese). (i) Zhangsan bei ta-de xuesheng ai de yao-si. Zhangsan BEI his student love DE want-die 'Zhangsan was loved by his students.'

Hence, it seems that the adding of the complement in BEI constructions may somehow cancel the requirement of the adversity reading on the subject. In the following discussion, the reader will see when examining BEI constructions, we consider both situations with and without the complement to avoid the controversy over this issue.

a. *Zhangsan bei ta-de xuesheng xinshang. Zhangsan BEI his student appreciate (1) 'Zhangsan was appreciated by his students.'

b. *Zhangsan bei ta-de xuesheng ai. 'Zhangsan was loved by his students.'

Zhangsan bei xuesheng da-shang-le.

Zhangsan BEI student hit-hurt-ASP (2)

'Zhangsan was hurt by the students.'

Corresponding Author: Pei-Jung Kuo, Department of Foreign Languages, College of Humanities and Arts, National Chiayi University, No.85, Wunlong Village, Minsyong Township Chiayi County 621, Taiwan Tel: (05) 2263411\#8218 Fax: (05) 2063072 


\section{J. Social Sci., 8 (3): 435-446, 2012}

However, this requirement in fact is not fully carried out if one examines the modern Chinese passives in the corpus. For example, according to some recent corpus studies by McEnery et al. (2003); McEnery and Xiao (2005); Xiao et al. (2006); Yang (2006) and Kuo (2010), it has been noticed that the adversity requirement does not hold tightly in Modern Chinese. These studies show that only $50-75 \%$ of the BEI passive has the adversity reading on the subject.

In the following discussion, we will first introduce the corpus works of Kuo (2010) and Peyraube (1989) which both address the issue of the adversity reading of the BEI passives. After that, we will point out that we may need to consider another historical factor when we examine the adversity reading change of the BEI passive. That is, in archaic Chinese, the BEI passive is not the only and major passive construction. We then investigate the WEI construction and conclude that in archaic Chinese, the WEI construction, as a major passive representative, also do not have a high percentage of adversity reading imposed on its subject. Further evidence from the comparison between the archaic and modern WEI...SUO passive also shows that the decline of the adversity reading is not limited to the BEI passive. These study results hence provide us with a new direction of the adversity reading decline of the BEI passive. We conclude the study in the last section.

\section{MATERIALS AND METHODS}

As mentioned previously, there are several corpus studies regarding the semantic change of the BEI passive. We presented two corpus studies: Kuo (2010) and Peyraube (1989). The former compares two sets of corpus data after 1900 to show that there is a decline of the adversity reading on the BEI passive. The latter traces the semantic change of the BEI passive back to the Archaic Chinese which is around 100 B.C. to 100 A.D. These two studies should give the readers an overview about the central issue of the adversity reading on the BEI passives.

Kuo (2010) compares two sets of Chinese data to see if there is indeed an adversity requirement on the subject in BEI constructions and if there is a gradual switch from the adversity/malificiary reading to the neutral or beneficiary reading. The basic information of her data is shown in Table 1.

The two sets of data are both collected from the political editorial sections of newspapers. The first set of data is from the book "XinMin Shou," a collection of political editorials written by Journalist and political activist Qichao Liang between 1902 and 1906 for the newspaper "XinMin CongBao" ("New Citizen Journal"). The word count of all his articles is around one hundred and twenty thousand. The second set of data is a collection of political editorials written by Journalist and political activist Hengwei Jin between 2008 and 2009 for the newspaper "The Liberty Times." The word count is around one hundred and twenty thousand, thus matching the first set of data.

The data were compared in the following ways: First, Kuo picked out all the BEI passives, including both long passives and short passives. Second, she examined all the VPs in the passive constructions and categorized them by the meaning of the verb and its complement, if any. Any repeated or unidentified verbs (i.e., typos) were excluded. She also made reference to the on-line Chinese dictionary by the Ministry of Education to determine whether the verb or the whole VP carried the adversity reading, since this can affect the subject (the link of the on-line dictionary website: http://dict.revised.moe.edu.tw/). The final results are shown in the first two columns in Table 2. Note that the same procedure was applied a second time to the two sets of data, but this time excluding any BEI constructions with complements. This step was taken to avoid the controversy about whether the complement may void the adversity requirement in BEI constructions (cf. Liu et al., 2001). The results are shown in the last two columns in Table 2.

\begin{tabular}{lll}
\multicolumn{3}{l}{ Table 1: The Basic Information of Data 1 and Data 2} \\
\hline Time & Data 1 & Data 2 \\
Author & 1902 1906 & 10/5, 2008 10/11, 2009 \\
Source & $\begin{array}{l}\text { Liang, Qichao } \\
\text { XinMin CongBao } \\
\text { (New Citizen Journal) }\end{array}$ & $\begin{array}{l}\text { Jin, Hengwei } \\
\text { The Liberty Times }\end{array}$ \\
$\begin{array}{ll}\text { Genre } \\
\text { Word count }\end{array}$ & $\begin{array}{l}\text { Political Editorial } \\
119,728\end{array}$ & 119,482 \\
\hline
\end{tabular}

Table 2: The Comparison Results of Data 1 and Data 2

\begin{tabular}{|c|c|c|c|c|}
\hline & Data 1 & Data 2 & $\begin{array}{l}\text { Data } 1 \\
\text { (with complement) }\end{array}$ & $\begin{array}{l}\text { Data } 2 \\
\text { (without complement) }\end{array}$ \\
\hline Total passive constructions & 22.000 & 94.000 & 21 & 69 \\
\hline $\begin{array}{l}\text { Valid passive constructions (excluding the repeated or } \\
\text { unidentified verb) }\end{array}$ & 16.000 & 80.000 & 15 & 60 \\
\hline Adversity VP & 14.000 & 61.000 & 14 & 45 \\
\hline Other VP & 2.000 & 19.000 & 1 & 15 \\
\hline Adversity VP Percentage & $87.500 \%$ & $76.250 \%$ & $93 \%$ & $75 \%$ \\
\hline The result of chi-square & $<.001$ & $<.001$ & & \\
\hline
\end{tabular}




\section{J. Social Sci., 8 (3): 435-446, 2012}

The results show that it is indeed true that the majority of the BEI passives have an adversity reading imposed on the subject, coming from the adversity VP. Moreover, the Chi-square test shows that there is a statistically significant drop in the number of passives with an adversity reading (regardless of whether the passive has a complement or not) between the early $20^{\text {th }}$ and $21^{\text {st }}$ centuries.

To sum up, the data presented so far support the initial observation that there has been a gradual decrease in the adversity reading on the subject. While it is true that the majority (around 75\%) of the BEI passive meet the adversity reading requirement on the subject, consistent with the statement about BEI constructions from Liu et al. (2001) Chinese textbook, it also appears that BEI constructions increasingly allow non-adversity VPs. The drop in the adversity reading requirement over the past hundred years is around $15 \%$ (one phenomenon is also worth noting: the emergence of the complement in BEI constructions has also increased in the past hundred years. For example, in Data 1 , the rate is 1 out of 16 $(6.25 \%)$. But in Data 2, the rate becomes 20 out of 80 (25\%). The use of different complements makes the BEI passives more dynamic, which adds a lot of variety in its expression ways).

The above study result shows that there is indeed a difference between our impression and the real reality of the BEI passive. In the literature, researchers hypothesize that the adversity reading may drop as the time goes by, but they differ in the cause of the dropping (see the discussions in Ma, 2003; Yang, 2006). There are two main hypotheses: one assumes that the dropping is due to language contact (i.e., Wang, 1957; Chao, 1968; Li and Thompson, 1981; Hashimoto, 1988) and the other assumes that the word BEI has been grammaticalized (i.e., Peyraube 1989; 2000; Tien, 2000; Xing, 2003; Yang 2006). The first hypothesis basically adopts the view of external influence, i.e., Language Contact. For example, Wang (1957) proposes that the dropping of the adversity reading of the BEI passive is because of language contact with the western languages. It may be the case that the BEI passive is influenced by the translation works (cf. Zhou and Feng, 1983; Wu, 1993; Zhu, 1996; Chen, 1997). And the May 4th movement in 1919 can be viewed as a dividing point. Other researchers support the view of internal change of the language itself (the second hypothesis). The word BEI experienced some internal change, shifting from a content word to a passive marker. For example, Hashimoto (1988) proposes that the adversity reading has declined from the end of the Tang Dynasty. Peyraube (1989) points out that the original BEI has the meaning of "blanket" as a noun and "to receive, to suffer" as a verb in the Han Dynasty. Because of the process of grammaticalization, BEI has become a preposition in the Sui-Tang Dynasty and even become a passive marker in modern Chinese.

Here we examine the study of Peyraube (1989) for more details. To support his proposal, Peyraube (1989) did a diachronic investigation of the BEI passive. For the database, he went back to the book of Shi Ji and Han Shu and searched all the sentences containing the word BEI. There are $149 \mathrm{BEI}$ in the main texts in the book of Shi Ji. BEIs in the footnotes are ignored. The search result shows that there are 56 nouns, which has the meaning of "blanket". There are also 88 verbal usage of BEI, which can be divided into different subcategories syntactically. When BEI is used as a transitive verb, it can either mean "to receive, to suffer" or "to cover, to wear". BEI can also be followed by a verb directly (similar to the structure of short passive) and means "to suffer, to be affected". Finally BEI can also emerge in a long passive structure which can mean either "to cover, to wear" or "to suffer, to be affected". This is shown in Table 3 (the total number of verbal BEI here is 80 . There are 8 verbal usage of BEI which are not discussed in Peyraube (1989)).

As shown in Table 3, Peyraube (1989) only found 11 tokens of sentences in which BEI has the meaning related to passive marker. He also indicated that it is not until the late Han Dynasty has the number of the passive meaning of BEI increased. For example, in the book of Han Shu, there are 309 BEIs in the main texts, excluding the footnotes. While there are 74 noun usage, there are 235 verbal usage. In the following five syntactic structures in Table 4, Peyraube (1989) did not give the accurate number for the first three structures in his paper. He only concluded that in the book of Han Shu, BEI's meaning "to receiver, to suffer" is slightly more numerous than the meaning "to cover, to wear" when BEI is used as a transitive verb. As for the short passive, the number is also remarkably increased. When it comes to the long passive structure, one can see that the number is still quite small.

Peyraube later also investigated the data from the medieval Chinese and concluded that the verbal BEI has become a preposition BEI in the Sui-Tang Dynasty. According to the above results, Peyraube (1989) proposes that BEI has undergone the process of grammaticalization (Li and Shi, 2006; Peyraube; 2000; Tien, 2000; Xing, 2003; Yang, 2006). Interesting, the process of grammaticalization does not apply to the word BEI only in the derivation of Mandarin. The same pattern has been attested by the BA construction in Mandarin as well, which has been proposed to share some syntactic similarities to the BEI passive (Huang et al., 2009). BA has also undergone grammaticalization from a verb to a preposition in the Sui-Tang Dynasty and currently functions as a disposal marker in modern Chinese (see Sun, 1996). 


\section{J. Social Sci., 8 (3): 435-446, 2012}

Although BEIs do emerge in the archaic Chinese as passive constructions, we believe that we should be more careful when examining the change of the adversity reading of the BEI passives. First of all, according to Table 3, there are only 149 sentences containing the word BEI in the book of Shi Ji. If we count only the verbal ones, we have 88 tokens. The meaning "to cover", to "wear" should be viewed as the beneficiary reading, while the one "to suffer", "to be affected" should be viewed as the adversity reading. In this way, this means that sixty percent of the BEI passive has the beneficiary meaning. This is quite strange compared to the recent corpus research conclusions that there is a "decline" of the adversity reading of the BEI sentences. One may argue that we should only concentrate on the BEI sentences which mean "to suffer" or "to be affected". In this way, the adversity reading should be $100 \%$ and it is possible that this requirement drops later. However, it should not be possible that there are only eleven passive sentences in a book like Shi Ji, which has more than fifty hundred and twenty thousand words.

Through the courses of archaic Chinese texts in our junior and high schools, we have learned that the passive construction is not limited to the BEI passive. Just like the cases we can use GEI, RANG, or JIAO as passive markers in modern Chinese, there are also several passive morphemes in archaic Chinese, such as WEI, JIAN, SHOU. We do use WEI as the major passive marker in the modern Chinese because in the Sui-Tang Dynasty, BEI somehow replaces WEI and becomes the major candidate for passive constructions (Chu, 1987; Peyraube 1989; Li, 1994; Wu, 2007). Take Fig. 1 for example, the rise and fall of various Chinese passives is summarized as follows (this figure is based on Chu (1987) and modified accordingly by other researchers' observations about Chinese passive constructions).

Table 3: The verbal BEI in the book of Shi Ji

\begin{tabular}{lll}
\hline The & The & $\begin{array}{l}\text { Sentence } \\
\text { number }\end{array}$ \\
meaning & structure & 21 \\
to receive, to suffer & $\mathrm{BEI}+\mathrm{NP}$ & 27 \\
to cover, to wear & $\mathrm{BEI}+\mathrm{NP}$ & 8 \\
to suffer, to be affected & $\mathrm{BEI}+\mathrm{V}$ & 21 \\
to cover, to wear & $\mathrm{BEI}+\mathrm{NP} 1+\mathrm{V}+(\mathrm{NP} 2)$ & 21 \\
to suffer, to be affected & $\mathrm{BEI}+\mathrm{NP} 1+\mathrm{V}+(\mathrm{NP} 2)$ & 3 \\
\hline
\end{tabular}

Table 4: The verbal BEI in the book of Han Shu

\begin{tabular}{lll}
\hline $\begin{array}{l}\text { The } \\
\text { meaning }\end{array}$ & $\begin{array}{l}\text { The } \\
\text { structure }\end{array}$ & $\begin{array}{l}\text { Sentence } \\
\text { number }\end{array}$ \\
\hline to receive, to suffer & $\mathrm{BEI}+\mathrm{NP}$ & $\mathrm{N} / \mathrm{A}$ \\
to cover, to wear & $\mathrm{BEI}+\mathrm{NP}$ & $\mathrm{N} / \mathrm{A}$ \\
to suffer, to be affected & $\mathrm{BEI}+\mathrm{V}$ & $\mathrm{N} / \mathrm{A}$ \\
to cover, to wear & $\mathrm{BEI}+\mathrm{NP} 1+\mathrm{V}+(\mathrm{NP} 2)$ & 20 \\
to suffer, to be affected & $\mathrm{BEI}+\mathrm{NP} 1+\mathrm{V}+(\mathrm{NP} 2)$ & 5 \\
\hline
\end{tabular}

\begin{tabular}{|c|c|c|c|c|c|c|c|c|c|}
\hline \multirow{2}{*}{$\frac{\mathrm{YU} / \mathrm{HU}}{\mathrm{JIAN}}$} & - & 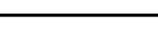 & & & & & & & \\
\hline & & & & & & & & & \\
\hline \multicolumn{10}{|l|}{ JIAN ... } \\
\hline \multicolumn{10}{|l|}{$\mathrm{YU} / \mathrm{HU}$} \\
\hline \multicolumn{10}{|l|}{ WEI } \\
\hline \multicolumn{10}{|l|}{ WEI ...JIAN } \\
\hline \multicolumn{10}{|l|}{ WEI...SUO } \\
\hline \multicolumn{10}{|l|}{ BEI } \\
\hline \multicolumn{10}{|l|}{ BEI ...SUO } \\
\hline \multirow{4}{*}{ Passive } & & & & Ending & Ending & Northern & Ending & & \\
\hline & Chou & Confucious & Mecius & & of & & of & & \\
\hline & Dymasty & & & Warring & Han & Southern & Tang & & \\
\hline & $1000 \mathrm{BC}$ & $500 \mathrm{BC}$ & & $000 \mathrm{AD}$ & & $500 \mathrm{AD}$ & $1000 \mathrm{AD}$ & $1500 \mathrm{AD}$ & Now \\
\hline
\end{tabular}

- : the time when the construction in questions is used as a major passive construction

--- : the time when the construction in question is used as a minor passive construction

Fig. 1: The historical derivation of Chinese passives 


\section{J. Social Sci., 8 (3): 435-446, 2012}

Therefore, we believe that if we want to track the semantic change of the BEI passive, our search should not be limited in the BEI passive only. Hence we propose that in determining whether there is a decline of the adversity reading in Chinese passives, we should examine the constructions involving these words as well. The hypothesis we would like to test is as follows: Apparently the word BEI doesn't serve as the main passive maker in archaic Chinese, but it may be influenced by the semantic requirement of other dominant passive constructions. Although most archaic passive words are not used in modern Chinese, it is still possible in the process of language internal change, the adversity reading of these disappeared constructions is carried over to the BEI passive gradually. Hence the percentage of the adversity reading in those archaic passive sentences is an important indicator to see if the adversity reading is already imposed in archaic Chinese passives. And to what extent can we relate the result to the explanation of the decline of the adversity reading in the BEI passive. We present the result of a pilot study and discuss the results in the following section.

\section{RESULTS}

We first present the search result from the HanDian Corpus and we discuss the results as follows. In the pilot study we focus on the morpheme WEI, which is a very common word for archaic passive construction. This is to follow Li (1994); Peyraube's (1989) and Wu (2007) proposal in that WEI passive is a major passive construction before the Sui-Tang Dynasty and is replaced by the BEI passive construction after this turning point. Hence to investigate the semantic content of the WEI passive in archaic Chinese should shed some light on the BEI passive in modern Chinese.

For the data source, we used the HanDian corpus created by the Academia Sinica (http://hanji.sinica.edu.tw/index.html)?. Note that WEI in fact has several meanings, including the meaning of "to be", "for", "in light of", "to do" and "passive marker". Examples are shown from (3-7) respectively (note that the tone of WEI (the second tone) cannot be used to differentiate the passive meaning from the others).

To be:

Taizi li, shi WEI Yanyi wong。

Prince establish, is WEI Yanyi king

'The prince is established. He is the King of yanyi.'
For:

Yu binke WEI chang ye yin。 With guests WEI long night drink

'Drink with guests for the long night.'

In light of:

Yiwei fei wo mo neng WEI ye。 Is not I not can WEI YE 'It is not what I can do.'

To do:

Sha ren fu zi er jun zhi, yu bu ren WEI。 Kill people father son then king it, I not bear WEI (6) 'To be a king, I kill people's fathers and sons. It is the thing that I cannot do.'

Passive marker:

Chen ge WEI qi zhu yong。

Courtiers each WEI one's master use

'Courtiers are used by their masters.'

In the following search results, those sentences containing WEI other than the meaning of being a passive marker are excluded. As one can see, although WEI has many meanings, the differences are quite distinct and most of the sentences containing WEI can be clearly distinguished. If there is a doubt, we use modern Chinese translation of the book Shi Ji to figure out whether WEI in question is used for the passive construction.

We used WEI as the keyword and ran the search in the HanDian Corpus. We then examined all the sentences containing WEI page by page. Sentences which do not contain the passive word WEI are all excluded. Due to the time limit, we stopped this process when we got fifty WEI passive sentences. Among these fifty tokens, we categorize their meaning by the following three labels: adversity/malificiary, neutral or beneficiary. If there is controversy, we made reference to the on-line Chinese dictionary by the Ministry of Education. The results are shown in Table 5.

As one can see, the adversity reading in the WEI passive though is dominant, is not an absolute requirement. Only $78 \%$ of the WEI sentences have adversity reading.

In addition to the categorization by meaning, we also categorize the fifty WEI sentences by their syntactic formats. The most well-known syntactic 


\section{J. Social Sci., 8 (3): 435-446, 2012}

categorization is to divide passives into long and short passives (Ting, 1998; Huang, 1999). The Agent emerges in the long passive, while it is absent in the short passive. The examples are shown in (8) and (9).

Long passive:

$\begin{array}{llrl}\text { Zhangsan bei } & \text { Lisi } & \text { da-le. } \\ \text { Zhangsan } & \text { BEI } & \text { Lisi } & \text { hit-ASP } \\ \text { 'Zhangsan } & \text { wass hit by Lisi.' } & \end{array}$

Short passive:

$\begin{array}{lll}\text { Zhangsan } & \text { bei } & \text { da-le. } \\ \text { Zhangsan } & \text { BEI } & \text { hit-ASP }\end{array}$

'Zhangsan was hit.'

In addition to the long and short difference, one thing that drew our attention is the co-occurrence of WEI and SUO in the WEI passives. In modern Chinese, we can still use WEI as the passive marker, but it has to appear with SUO. Without SUO, the sentence is judged as ungrammatical, as shown in (10) (in modern Chinese, SUO is optional in the BEI passive (Chiu, 1993; Ting, 2003)). This WEI... SUO construction in modern Chinese usually emerges in the literary texts such as newspaper or news reports.

\section{Zhangsan WEI Lisi *(SUO) qipian. Zhangsan WEI Lisi (SUO) cheat. 'Zhangsan was cheated by Lisi.'}

Hence what we did next is to divide the WEI passives into the ones without SUO and the ones with SUO. Both subgroups are categorized by their syntactic formats as long or short passives. The results are shown in Table 6.

For the sentences without SUO, we found more long passives than short passives. For the other sentences with SUO, we only found long passives.

Semantically, we further investigated the WEI sentences with SUO and found out that these 22 sentences have adversity reading only (this observation does not hold for the WEI...SUO construction in modern Chinese. See discussions below. The contrast is shown below).

Bi Wuxu fu xiong WEI lu yu chu。 He Wuxu father brother WEI kill in Chu 'Wuxu his father and brothers were killed in Chu'
Table 5: The WEI passive

\begin{tabular}{llc}
\hline Sentence Meaning & Sentence Number & Percentage \\
\hline Adversity/Malificiary & 39 & $78 \%$ \\
Neutral & 10 & $20 \%$ \\
Beneficiary & 1 & $2 \%$ \\
\hline
\end{tabular}

Table 6: The WEI...SUO passive

\begin{tabular}{|c|c|c|c|c|}
\hline \multirow{2}{*}{$\begin{array}{l}\text { Syntactic } \\
\text { construction }\end{array}$} & \multicolumn{2}{|c|}{$\begin{array}{l}\text { WEI } \\
\text { without SUO }\end{array}$} & \multicolumn{2}{|c|}{$\begin{array}{l}\text { WEI } \\
\text { with SUO }\end{array}$} \\
\hline & Number & Percentage & Number & Percentage \\
\hline Long & 17 & $34 \%$ & 22 & $44 \%$ \\
\hline Short & 11 & $22 \%$ & 0 & $0 \%$ \\
\hline
\end{tabular}

Yan zai ye nan, sui WEI tainxia hao。

Yan is Ye south thus WEI people call (12)

'Yan is in the south of Ye, thus it was called its name by people.'

Jia pin, WEI ren SUO lue mai。

Family poor, WEI people SUO kidnap sell (13) 'The family is poor, (she) was kidnapped and sold by someone.'

As shown in (11), this passive has the adversity reading since the main verb is "kill". On the other hand, the verb in (12) is "call", hence it has the neutral reading. The one in (13) is the WEI...SUO is "kidnap" and "sell", hence it has the adversity reading. For the full list of all the WEI...SUO sentences, please see Appendix 1 for more details.

To summarize, based on the above preliminary investigation, we have the following three generalizations. First, among the fifty tokens, we found that the WEI passive is not restricted to adversity reading. But the adversity reading is still dominant. Secondly, the emergence of SUO is strongly connected to the appearance of the Agent. That is, it only occurs in the long passive. Thirdly, when WEI emerges with SUO, the sentence is restricted to the adversity reading.

\section{DISCUSSION}

The WEI passive: The first result shows that only $78 \%$ of the WEI sentences have adversity reading. It has the following implications. First of all, this result further confirms that the BEI sentences are not the majority of the archaic passive. Other words such as WEI constitute most part of the passive family (i.e., Fig. 1). Furthermore, the fifty tokens of the WEI passive only occupies a very small part of the book of Shi Ji, which indicates that the usage of passive is definitely not that 'rare'. Secondly, the adversity reading percentage is not very high in the WEI passives. The number $78 \%$ means that the majority of the WEI passives has the adversity reading, but it is not an absolute requirement. 
Appendix 1: Twenty-two WEI...SUO sentences in Archaic Chinese

1. Source
Archaic Chinese
Modern Chinese
Translation
2. Source
Archaic Chinese
Modern Chinese
3. Source
Archaic Chinese
Modern Chinese
Translation
4. Source
Archaic Chinese

Modern Chinese Translation

5. Source Archaic Chinese

Modern Chinese Translation

6. Source

Archaic Chinese

Modern Chinese Translation

7. Source

Archaic Chinese

Modern Chinese

Translation

8. Source

Archaic Chinese

Modern Chinese

Translation

9. Source

Archaic Chinese

Modern Chinese

Translation

10. Source

Archaic Chinese shi ji juan qi xiang yu di qi

xiang ji zhe, xia xiang ren ye, zi yu chu qi shi, nian er shi si liang, liang fu ji chu jiang xiang yan, WEI qin jiang wang jian SUO lu zhe ye 。 Xiang-ji-de shufu shi Xiang-liang, Xiang-liang-de fuqin shi Xiang-yan, jiushi bei Qin jiang Wang-jian SUO shahai de nawei chuguo dajiang 。 shi ji juan ba gao zu ben ji di ba zhu shi shen qiu dao li ji yun "wang-mang yuan cheng ren, ju jin he ce, zu fu fen mu WEI shui SUO chong, yin he ru shen chuan, ci wang mang he yin ku ye 。 Wang-mang shi Yuan-cheng ren, zhuzai kaojin hean bian, zufu-de Translation fenmu bei shui chonghui 。

shi ji qin shi huang ben ji di liu

er shi jiu nian, shi huang dong you 。zhi yang wu bo lang sha zhong, WEI dao SUO jing 。 qiu fu de, nai ling tian xia da SUO shi ri 。

Er-shi-jiu nian (qian 218, Shi-huang dao dongfang qu xunyoun D Daod Yang-wu-

xian Bo-lang-sha shi, zaodao Zhang-liang he yi-ming lishi-de xingci, cike wuzhong fuche, Shi-huang shoule jingxia, zhuona cike meiyou zhuodao, jiu mingling quanguo da guimo soubu-le shitian 。

shi ji qin shi huang ben ji di liu zhu shi

fang shi xu shi deng ru hai qiu shen yao, shu sui bu de, fei duo, kong qian, nai zha yue: p peng lai yao ke de, ran chang WEI da jiao yu SUO ku, gu bu de zhi, yuan qing shan she yu ju, jian ze yi lian nu she zhi 」」 shi huang meng yu hai shen zhan, ru ren zhuang 。

Fangshi Xu-fu dengren dao haizhong xunzhao shenyao, hao ji-nian dou meiyou zhaodao, yongqu henduo qiancai, kongpa zaoshou qianze, ushi dui Shi-huang shuo: "Peng-lai-xian-dao-de shenyao shi keyi nadao-de, raner hangxing shi changchang bei da shayu xiji, suoyi wufa daoda, xiwang pai xie shanyu shesha shayu-de ren he women yiqi qu, shayu chuxian jiu yong da gongjian shesha ta 。 "Shi-huang mengjian ziji he haishen zhandou, haishen de xingzhuang haoxiang ren shi ji wai qi shi jia di shi jiu

dou huang hou xiong dou zhang jun, di yue dou guang guo, zi shao jun 。 shao jun nian si wu sui shi, jia pin, WEI ren SUO lue mai, qi jia bu zhi qi chu yu jia, zhi yi yang, WEI qi zhu ru shan zuo tan, mu wo an xia bai yu ren, an beng, jin ya sha wo zhe, shao jun du de tuo, bu si 。

Dou huanghou-de gege Jiaozuo Dou Zhang-jun, didi Jiaozuo Dou Guang-guo, ingzi Shao-jun。Didi

zai si, wu-sui shi bei ren youguai maidiao, ta bu zhidao jia zai nail, ta bei daidao Yiyang, bang ta-de zhuren dao shan shang kanchai, huanghun shi gaoda shang bai ren zai nali kanchai, jieguo shan shang bengta le, yasi le xuduo ren, zhiyou shao jun taozou mei si 。

shi ji qin ben ji di wu zhu shi

zhou tong xing SUO feng, zuo chuan yun yu, guo, jiao, hua, huo, yang, han, WEI jie ji xing ye 。 du yu yun ba guo jie WEI jin SUO mie 。

Zhou-chao feng tongxing WEI hou, Zuo-chuan shuo yu guo

jiao, hua, huo, yang, han, WEI zhe-xie guojia dou shi ji xing。Du yuyan zhe ba-ge guojia dou hui bei Jin-guo miewang 。

shi ji ji bu luan bu lie chuan di si shi

shi liang wang peng yue WEI jia ren shi, chang yu bu you, qiong kun, lin yong yu qi, WEI jiu ren bao 。 shu sui, peng yue qu zhi ju ye zhong WEI dao, er bu WEI ren SUO lue mai, WEI nu yu yan 。

Luan-bu shi Liang-di ren。 Dangchu Liang wang Peng-yue zuo pingmin-de shihou

cengjing he Luan-bu jiaowang。 Luan-bu jiali pinkun, zai qi di bei ren guyong, ti maijiu-de renjia zuo yonggong 。 Guo-le ji-nian, Peng-yue laidao Ju-ye zuo qiangdao, er Luan-bu que bei ren qiangxing jiechi chumai, dao Yan di qu zuo nupu 。

shi ji huai yin hou lie chuan di san shi er

xin fang zhan, yue: 「wu hui bu yong kuai tong zhi ji, nai WEI er nu zi SUO zha, qi fei tian zai!」 sui yi xin san zu 。

Han-xin lin zhan shi shuo: "Wo houhui meiyou caina Kuai-tong-de jimou, yizhi bei funu xiaozi SUO qipian, nandao bushi tianyi ma "Yushi zhusha-le Han-xin san zu。 shi ji feng chan shu di liu

zi zhou ke yin hou shi si shi, shi yi shuai, li le fei, zhu hou zi xing, er you wang WEI quan rong SUO bai, zhou dong xi luo yi 。

Shidao gengjia shuairuo, liyue gengjia feiqi, zhuhou ziyi xing shi, er Zhou-you-wang

bei quan rong dabai 。Zhou chao douching dong qian dao Luo-yi 。

shi ji qin ben ji di wu

xiang gong er nian, rong WEI quan qiu, shi fu ji zhi, WEI rong ren SUO lu。 sui yu, fu gui shi fu。 


\section{J. Social Sci., 8 (3): 435-446, 2012}

Modern Chinese

11. Source

Archaic Chinese

Modern Chinese

Translation

12. Source

Archaic Chinese

Modern Chinese

Translation

13. Source

Archaic Chinese

Modern Chinese Translation

14. Source

Archaic Chinese

Modern Chinese

Translation

15. Source

Archaic Chinese

Modern Chinese

Translation

16. Source

Archaic Chinese

Modern Chinese

Translation

17. Source

Archaic Chinese

Modern Chinese

Translation

18. Source

Archaic Chinese

Modern Chinese

Translation

19. Source

Archaic Chinese

Modern Chinese Translation

20. Source

Archaic Chinese

Modern Chinese

Translation

21. Source

Archaic Chinese

Modern Chinese Translation

22. Source

Archaic Chinese

Modern Chinese

Translation
Xiang gong er nian (qian 776), Xi-rong baowei Quan-qiu, wo fuqin qu gongda Translation Xi-rong, dan bei Xi-rong fulu。

shi ji feng chan shu di liu

shi huang feng chan zhi hou shi er sui, qin wang zhu ru sheng ji qin fen shi shu, zhu lu wen xue, bai xing yuan qi fa, tian xia pan zhi, jie e yue: qin $_{\text {shi huang }}$ shang tai shan, WEI bao feng yu SUO ji, bu de feng chan 。」

Shi-huang fengchan yihou shi-er nian, Qin chao miewang。Na-xie rusheng-men jihen

Qin chao fenhui shishu, tusha、 wuru wenxue shiren, baixing yuanhen Qin chao falu, tianxia ren dou beipan Qin chao, yiner dou echuan shuo: 「Shi-huang shang Tai-shan, bei baofengyu SUO zu, mei neng xing fengchan li 。

shi ji zhou ben ji di si zhu shi

di wang shi ji yun: $\lceil$ ming dan o sui ju tian zi zhi WEI hao, WEI zhu hou zhi SUO yi bi, yu jia ren wu yi 。」

Zai diwang shiji pian ceng shuo: $\lceil$ Suiran weiju tianzi-de minghao,

dan shiji shang bei zhuhou bipo zuoshi, qishi yu pingmin meiyou liangyang。」

shi ji qin ben ji di wu

zhang yi fa shu, shu wang kai zhan bu sheng, WEI yi SUO mie ye 。

Zhang-yi taofa Shu guo, Shu guo zhanbai, bei Zhang-yi xiaomie diao

shi ji zhou ben ji di si

u shi long li yao zi, WEI ren SUO shou, bao ren na zhi yu wang, gu yue bao si

Zhe-ge ren-de nuer shi Long-li yaonie de zisi, bei ren shoufu-le, Bao

ren jiang ta xian gei huang di, ming jiao Bao-si 。

zhou ben ji di si zhu shi

du yu yun hua guo dou fei, he nan gou shi xian, WEI qin SUO mie, shi shu zheng, jin, hou shu zhou 。

Du-yun-hua guodou Fei, ye jiushi Henan fu-de gou shi xian, hui

bei Qin xiaomie, benlai shuyu Zheng yu Jin guo, houlai shu yu Zhou guo 。

shi ji xia ben ji di er zhu shi

zhou wu wang feng yu hou yu qi, hao dong lou gong, er shi yi dai WEI chu SUO mie 。

Zhou-wu-wang jiang yu-de houdai cefeng zai Qi zhe yi-dai, chengzuo

Dong-lou-gong, dao-le di er-shi-yi dai bei Chu guo miediao 。

shi ji xia ben ji

zhuo sha yi, peng zhi, yi shi qi zi, zi bu ren shi, sha yu qiong men。 zhuo yin yi shi, sheng jiao ji yi 。 shi jiao mie zhen guan shi ji zhen xun shi, er xiang WEI jiao SUO mie, hou min gui yu you reng, sheng shao kang 。

Han-zhuo sha-le Hou-yi zhihou, zhulai WEI Hou-yi-de erzi, ta bu gan chi, er

zai Diong-men zisha 。 Han-zhuo qiangzhan Hou-yi-de qishi sheng-le liang-ge erzi, yi-ge jiao Yun-jiao, ling yi-ge jiao Yun-yi 。 Han-zhuo yao sha Xia-di-xiang, bian mingling Jiao xiaomie Zhen-guan-shi ji Zhen-xun-shi, er dou bei Jiao xiaomie-le 。Xiang de feizi Houmin dangshi huaiyun, taodao niangjia You-reng-shi buluo (Shan-dong Ji-nan), shengxia Shaokang

shi ji xia ben ji di er

pan yu you tian, bu xu min shi, WEI yi SUO zhu, bu de fan guo

Pan-yu zai yewai wanle, bu neng tixu minjian jiku, bei Hou-yi quzhu, bu

neng fan hui guojia dang wang 。

shi ji WEI kang shu shi jia di qi

qing hou er shi nian, zhou you wang yin luan, WEI quan rong SUO shi 。

Zhou er-shi nian, Zhou-you-wang yinluan bei Quan-rong shahai 。

shi ji qin ben ji di wu

jiu yue ren $\mathrm{xu}$, yu jin hui gong yi wu he zhan yu han di。 jin jun qi qi jun, yu qin zheng li, hai er ma jing o miao gong yu hui xia chi zhui zhi, bu neng de jin jun, fan WEI jin jun SUO WEI 。

Jiu yue ren xu ri, yu Jin-hui-gong Yi-wu zai Han di jiaozhan。 Jin jun shuaixia ziji-de budui duzi wang qian chong, gen Qin jun zhengdou $。$ Miao gong zhui shang qian, danshi zhuo bu dao Jin jun, ziji que bei Jin jun weizhu。

shi ji xiang yu ben ji di qi

wu wen xian ji zhi ren, hou ze WEI ren SUO zhi 。

Wo tingdao-le jiu xian fa zhi ren, man-le jiu hui bei ren kongzhi 。

shi ji xiang yu ben ji di qi zhu shi

liu, zheng yi ye hou WEI chen SUO bing, gu yue chenliu 。

Liu, shi Zheng guo-de shoudou。Houlai bei Chen guo hebing, gaiwei, Chen guo-de shou dou-Chen Liu 。 


\section{J. Social Sci., 8 (3): 435-446, 2012}

This is similar to the conclusion of the BEI passive in modern Chinese, based on several corpus research results (50-75\%). If this conclusion is on the right track, this may indicate that the adversity requirement, at least in the archaic WEI passive and the modern BEI passive constructions, is never a $100 \%$ requirement. As long as more than half of the passives have the adversity reading, we accept the impression of such a requirement imposed on passives and our textbook of language teaching.

Lastly, the adversity reading percentage of the WEI passive and the BEI passive do not show a dramatic difference. We have shown that the representative of the passive construction in archaic Chinese is the WEI passive and the representative in modern Chinese is the BEI passive. In other words, the difference of passive constructions throughout the time only lies in the different morpheme that we use mostly in the passive constructions. In archaic passive it is WEI and in modern Chinese it becomes BEI. While the former has $78 \%$ of adversity passive, the latter has around $50-75 \%$ adversity passive. The result of the current pilot study sheds light on the hypothesis that it is possible that the semantics of the BEI passive can be influenced by the WEI passive. We can see that the adversity reading percentage of the BEI passive $(75 \%$, the highest number in one of the current corpus studies) is very close to the one of the archaic WEI passive (78\%). It is possible that when the BEI passive replaces the WEI passive (Li, 1994; Peyraube, 1989; Wu, 2007) and therefore its semantic distribution is similar to one of the WEI passive. Later the adversity reading of the BEI passive may change according to other historical factors. Hence it can also be as low as around 50\% (the lowest number in one of the current corpus studies).

The WEI...SUO passive: Our second research result shows that the WEI...SUO construction is only compatible with long passive construal. That is, the WEI...SUO construction must come with an Agent. There is a quite natural syntactic explanation for this. As proposed by Ting (1998) and Huang (1999), the long passive involves A'-movement, while the short passive involves A-movement. According to Huang (1999) and Ting (1998), the structure of long BEI passive is shown in (14). The Operator undergoes A'movement and adjoin to the lower IP. It then co-indexes a base-generated NP at the higher IP.

$\left[{ }_{\text {IP }}\right.$ Zhangsan $_{\mathrm{i}}\left[{ }_{\mathrm{VP}}\right.$ BEI $\left[{ }_{\mathrm{IP}} \mathrm{OP}_{\mathrm{i}}\left[{ }_{\mathrm{IP}}\right.\right.$ Lisi $\left[\mathrm{vP}_{\mathrm{VP}}\right.$ kill $\left.\left.\left.\left.\left.-\mathrm{ASP}_{\mathrm{i}}\right]\right]\right]\right]\right]$

Wu (2007) has argued that when BEI passive replaces the BEI passive, their syntactic structures do not differ from each other. This is shown in (15).

The passive structure derivation from WEI to BEI construction: (Wu 2007):

$$
\begin{aligned}
& \text { WEI copulaconstruction long WEI passive long BEI passive } \\
& \mathrm{WEI}_{\text {copula }}+\left[\mathrm{N}_{\text {possessor }}+\mathrm{N}\right]_{\mathrm{NP}} \rightarrow \underset{\text { by reanalysis }}{\mathrm{WEI}_{\text {passive }}+\left[\mathrm{N}_{\text {agent }}+\mathrm{V}\right]_{\mathrm{S}} \rightarrow \mathrm{BEI}_{\text {passive }}+\left[\mathrm{N}_{\text {agent }}+\mathrm{V}\right]_{\mathrm{S}}} \text { by analogy }
\end{aligned}
$$

Hence we assume that the syntactic structure proposed for the BEI passive should be applicable for the WEI passive in archaic Chinese, which is shown in (16):

$$
\left[{ }_{\text {IP }} \text { Zhangsan }_{\mathrm{i}}\left[_{\mathrm{VP}} \mathrm{WEI}\left[{ }_{\text {IP }} \mathrm{OP}_{\mathrm{i}}\left[_{\mathrm{IP}} \text { Lisi }\left[{ }_{\mathrm{VP}} \text { kill }-\mathrm{ASP}_{\mathrm{i}}\right]\right]\right]\right]\right]
$$

Chiu (1993) and Ting (1999) propose that the particle SUO can only show up in the chain of A'-movement. Hence in Chinese, only relative clause and the long passive allow the emergence of SUO, as shown in (17) and (18):

Relative clause:

$\begin{array}{lllll}\text { Zhangsan } & \text { (suo) } & \text { xihuan } & \text { de } & \text { nusheng } \\ \text { Zhangsan } & \text { (SUO) } & \text { likes } & \text { DE } & \text { girl }\end{array}$

'The girl who Zhangsan likes.'
Long passive:

Zhangsan bei Lisi (SUO) wujie Zhangsan BEI Lisi (SUO) misunderstanding 'Zhangsan was misunderstood by Lisi.'

It is therefore not surprising that we only found the WEI...SUO construction in the format of long passive. We have seen that the WEI...SUO construction also involve A'-movement like its modern BEI...SUO counterpart. And the particle SUO is only compatible with A'-movement. Hence we should expect to see WEI...SUO construction only with long passives.

Our third result gives us something interesting to be investigated in the future. It shows that all WEI...SUO sentences have an adversity reading. Since our sample is small, we are not sure whether it is just a coincident that all the WEI...SUO sentences have to have the adversity reading. Let us assume that the 443 


\section{J. Social Sci., 8 (3): 435-446, 2012}

adversity reading in archaic Chinese is relatively high (presumable $100 \%$ in our current data). With this assumption, we discuss the semantic comparison between the archaic and modern WEI...SUO constructions and provides a possible explanation for the adversity reading in the WEI...SUO construction from a syntactic perspective.

Semantically, there seems to be evidence showing in modern Chinese, the WEI...SUO construction does not have a high percentage of Adversity reading. For example, Xiao et al. (2006) searched the Lancaster Corpus of Mandarin Chinese (i.e., LCMC) built by McEnery et al. (2003), in which the content is the written texts in the early 90s in Mainland China. The result of the WEI...SUO construction is shown in Table 7 (to make the comparison more accurate, the same genre of written text should be selected. That is, we should also examine the WEI...SUO construction in historical written text. Here the adoption of the LCMC may lead to different result. But since our point here is to show that there may be a decline of the Adversity reading in the WEI...SUO construction as well. We will simply assume that these two pieces of data are comparable).

If one assumes that there is a very high percentage of adversity reading in the archaic WEI...SUO passive, there seems to be a dramatic decline of this reading in modern Chinese. Apparently there is adversity reading dropping in the WEI...SUO construction. The result of the chi-square test also shows that there is a significant gap between the archaic WEI...SUO construction and the modern WEI...SUO construction $(p<.001)$. This is not hard to imagine, since it is quite easy for native speakers to come up with a WEI...SUO sentence with non-Adversity reading, as shown in (19) and (20):

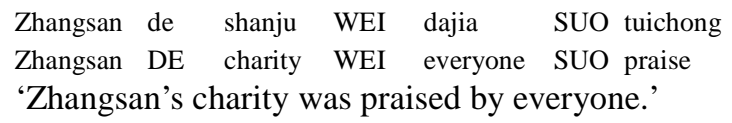

Xiaohua WEI xiaoming de zhenqing SUO gandong

Xiaohua WEI xiaomng DE truefeeling SUO touch (20)

'Xiaohua was touched by xiaoming's true feelings.'

Syntactically, we would like to provide a structural account to explain why adversity reading is co-related to the WEI...SUO construction. Tsai (2008), following Pylkkanen (2001; 2008), proposed that there is an Applicative Projection [ApplP] between $v \mathrm{P}$ and VP based on Huang's and Ting's passive structure to explain the existence of the adversity requirement in Chinese. The ApplP can only be projected if there is a negative event denoting VP. After adding the ApplP to the structure (14), the structure for the long passive should be like the one in (21). On its the way to the lower adjoined IP position, the Operator has to stop at Spec, ApplP to receive the adversity theta-role form the ApplP head, which then explains the source of the adversity reading in the passive. The trace at Spec, ApplP can be overtly realized as SUO, as shown in (22):

$$
\begin{aligned}
& \left.\left[{ }_{I P} \text { Zhangsan }_{\mathrm{i}}\left[{ }_{\mathrm{VP}} \text { BEI [ }{ }_{I P} \mathrm{OP}_{\mathrm{i}}\left[_{I P} \operatorname{Lisi}\left[{ }_{\mathrm{vP}} \mathrm{t}_{\mathrm{i}}\left[_{\mathrm{ApplP}} \mathrm{t}_{\mathrm{i}}\left[{ }_{\mathrm{VP}} \text { kill }- \text { ASP }_{\mathrm{i}}\right]\right]\right]\right]\right]\right]\right]
\end{aligned}
$$

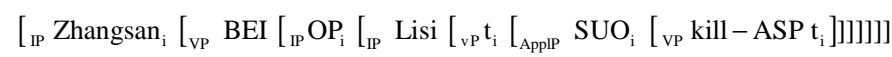

As proposed above, the structure of the BEI passive may be applicable to archaic WEI passive. Recall that in the archaic WEI passive, when SUO emerges, the whole passive sentence must have an adversity reading. We have proposed that SUO is an overtly realization of the Operator trace in (22). This means when SUO emerges, the Operator must stop at
Spec, ApplP before moving on. The lexical item SUO then has to take the adversity theta-role from the ApplP head. This then explain the obligatory adversity in the WEI...SUO construction. On the other hand, if the WEI passive has an adversity reading but comes without SUO, this indicates that the trace is not overtly realized in (23):

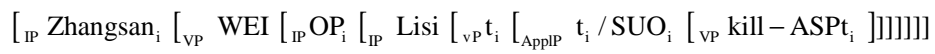

As for the WEI passive without the adversity reading, since there is no negative event denoting VP, there is no
ApplP, as shown in (24). This is the construction for those WEI passives without the adversity reading.

$$
\left.\left.{ }_{\text {IP }} \text { Zhangsan }_{\mathrm{i}}\left[_{\mathrm{VP}} \text { WEI }\left[{ }_{I P} \text { OP }_{\mathrm{i}}\left[_{\text {IP }} \text { Lisi }\left[_{\mathrm{VP}} \mathrm{t}_{\mathrm{i}}\left[_{\mathrm{VP}} \text { kill }- \text { ASP } \mathrm{t}_{\mathrm{i}}\right]\right]\right]\right]\right]\right]\right]
$$




\section{J. Social Sci., 8 (3): 435-446, 2012}

Table 7: WEI...SUO in modern Chinese

\begin{tabular}{|c|c|c|c|c|c|c|c|}
\hline \multirow[t]{2}{*}{ Passive Type } & \multirow[t]{2}{*}{ Passive Marker } & \multicolumn{2}{|l|}{ Negative } & \multicolumn{2}{|l|}{ Neutral } & \multicolumn{2}{|l|}{ Positive } \\
\hline & & Frequency & Percent & Frequency & Percent & Frequency & Percent \\
\hline Syntactic Passive & WEI...SUO & 13 & $18.9 \%$ & 27 & $39.1 \%$ & 29 & $42.0 \%$ \\
\hline
\end{tabular}

Note that in modern Chinese, the WEI...SUO construction is not limited to adversity reading. The above dichotomy is not applicable to the modern WEI...SUO construction. One possibility is that the construction in (24) is suspended and it is replaced by a neutral XP in the position between $v \mathrm{P}$ and VP. This

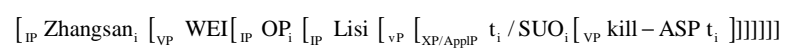

In short, our pilot study also shows that in addition to the adversity reading dropping in the BEI passive, the same phenomenon can also be observed in the WEI...SUO construction. This indicates that although the WEI...SUO construction is not very common in the passive family in modern Chinese, it still shows the decline of the adversity reading, which means the adversity reading dropping is not a single phenomenon in the BEI passive. This further supports our proposal that when we investigate the adversity reading decline in the BEI passive, we should consider other passive constructions as well. The historical complexity and change may lead us to a different conclusion.

\section{CONCLUSION}

To summarize, this study investigates the historical change of passive constructions and questions the proposal that the adversity reading decline of the BEI passive can be limited to this construction alone. We propose that other passive constructions, especially the WEI construction, should be investigated as well. Our pilot study shows that this is indeed the right direction to go. The results show that the main passive construction in archaic Chinese: the WEI construction, does not hold a very high adversity reading. In addition, if we focus on the WEI...SUO construction, it also shows the decline of the adversity reading. Last, the current syntactic analysis for the BEI...SUO construction can be applied to the WEI...SUO construction, which provides further evidence of the corelation of these two constructions.

Unavoidable, there is a glitch of the results presented above. Since we only focus on a small amount of data, the final result may be different from what we have presented. But because of the pilot study, we believe that our research hypothesis is worth testing. Hence what we need to do next is the carry out a full examination of the WEI construction in the book of Shi Ji and to extend the means that for the modern passive, there is only single long passive construction. It is just the case that the projection between $v \mathrm{P}$ and VP can be either an XP or an ApplP, as shown in (25). Hence when SUO is overtly realized in (25), we do not always have a corresponding adversity reading.

same procedure to other archaic passive constructions as well. All the results will benefit us in the understanding of the BEI construction and other passive constructions in their historical semantic changes.

\section{ACKNOWLEDGEMENT}

This study is part of the research project sponsored by the National Science Council, Taiwan (Grant No. NSC 98-2410-H-415-046). We hereby acknowledge the financial support of the NSC. The author would also like to thank Professor Ting-Hui Wen and the two anonymous reviewers for their comments and suggestions on the previous version of this paper. All errors remain ours.

\section{REFERENCES}

Chao, Y.R.., 1968. A Grammar of Spoken Chinese. 1st Edn., University of California Press, ISBN-10: 0520002199 , pp: 847.

Chen, D.A., 1997. English and Chinese translation: A comparative study.

Chiu, B.H.C., 1993. The Inflectional Structure of Mandarin Chinese. University of California, Los Angeles, pp: 480.

Chu, C.C.H., 1987. Historical Syntax-Theory and Application to Chinese. Crane Publisher, pp: 237.

Hashimoto, M.J., 1988. The Structure and Typology of the Chinese Passive Construction. In: Passive and Voice, Shibatani, M., (Ed.). John Benjamins Publishing Company, Amsterdam, pp: 329-354.

Hsin, A.L.C., 1990. The formation and function of passive in Mandarin Chinese and english: A contrastive analysis (I, II). English Teac. Learn., 15: 76-83.

Huang, C.T.J., 1999. Chinese passives in comparative perspective. Tsing Hua J. Chinese Stud., 29: 423509. 
Huang, C.T.J., Y.H.A. Li and Y. Li, 2009. The Syntax of Chinese. 1st Edn., Cambridge University Press, ISBN-10: 052159958X, pp: 404.

Kuo, P., 2010. Revisiting Chinese passives. Poster presented at the 84th Linguistic Society of America (LSA) Annual Meeting, San Francisco.

Li, C. and Y. Shi, 2006. Grammaticalization Foundation and Syntactical Environment of Passive Marker 'Jiao'] Guhanyu yanjiu.

Li, C.N. and S.A. Thompson, 1981. Mandarin Chinese: A Functional Reference Grammar. 1st Edn., University of California Press, ISBN-10: 0520042867, pp: 691.

Li, S., 1994. The study of the bei-construction in Modern Chinese. Peiking University Press.

Liu, Y., W. Pan and W. Gu, 2001. Practical modern Chinese grammar, Revised Edition [Shiyong Xiandai Hanyu Yufa (Zengdi Ban)]. Shangwu Publisher.

Ma, Q., 2003. Cihui Yufahua De Dongyin. [Motivation of Grammaticalization in vocabulary]. Hanyu xuexi, 2(N15:20).

McEnery, A. and R.Z. Xiao, 2005. Passive constructions in English and Chinese: A corpusbased contrastive study. Languages Contrast, 6: 109-149. DOI: 10.1075/lic.6.1.05xia

McEnery, A., Z. Xiao and L. Mo, 2003. Aspect marking in English and Chinese: Using the Lancaster corpus of Mandarin Chinese for contrastive language study. Literary Linguistic Comput., 18: 361-378. DOI: 10.1093/1lc/18.4.361

Peyraube, A., 1989. History of the passive constructions in Chinese until the 10th century. J. Chinese Linguistics, 17: 335-371.

Peyraube, A., 2000. Westernization of Chinese grammar in the 20h century: Myth or reality. J. Chinese Linguistics, 28: 1-25.

Pylkkanen, L., 2001. What applicative heads apply to. Proceedings of the 24th Annual Penn Linguistics Colloquium, University of Pennsylvania, Philadelphia, pp: 197-210.

Pylkkanen, L., 2008. Introducing arguments. MIT Press.

Sun, C., 1996. Transitivity, the BA construction and its History. J. Chinese Linguistics, 23: 159-195.
Tien, Z.P., 2000. A diachronic and synchronic study on the BEI passive in Mandarin Chinese. (Unpublished MA thesis). Fu Jen Catholic University.

Ting, J., 1998. Deriving the BEI-construction in Mandarin Chinese. J. East Asian Linguistics, 7: 319-354. DOI: 10.1023/A:1008340108602

Ting, J., 2003. The Nature of the Particle SUO in Mandarin Chinese. J. East Asian Linguistics, 12: 121-139. DOI: 10.1023/A:1022463417943

Tsai, W.T.D., 2008. On Middle Applicatives. Talk given at the Ling Lunch Presentation in University of Connecticut.

Tseng, C.C., 2004. You Pinghengyuliaoku He Zhongjieyu Yuliao Kan Hanyu Beiziju Biaoshu De Wenhua Yiyun. [Cultural representations of the passive voice and the Mandarin Bei-construction in L1 and L2 corpora]. J. Chinese Language Comput., 15: 89-101.

Wang, L., 1957. Hanyu beidongshi de fazhan [The development of the passive in Chinese]. Yuyanxue Luncong, 1: 1-16.

Wu, C.C., 1993. Chinese-English translation through contrastive Analysis. Crane Publisher.

Wu, Y., 2007. The early history of the Chinese passive. (Unpublished Doctoral Dissertation). University of Stanford.

Xiao, Z.R., A. McEnery and Y. Qian, 2006. Passive constructions in English and Chinese: A Corpusbased Contrastive Study. Languages Contrast, 6: 109-149. DOI: 10.1075/lic.6.1.05xia

Xing, J.Z., 2003. Grammaticalization of verbs in Mandarin Chinese. 1st Edn., LINCOM Europa, ISBN-10: 3895867551

Yang, H.L., 2006. Grammaticalization of the Chinese Morpheme BEI: Using Diachronic/Synchronic Corpora. Arizona State University. pp: 194.

Zhou, Z. and W. Feng, 1983. Comparison Between Chinese and English Passive Sentences and Discussion on Methods of Translation. Language Learning and Communication, 2: 309-321.

Zhu, C., 1996. Syntactic status of the agent: Its Significance for information presentation in translating the passive between Chinese and English. Multilingua, 15: 397-418. DOI: 10.1515/mult.1996.15.4.397 